\title{
Effects of COVID-19 on national tuberculosis elimination programme strategies during March to May 2020 on Tumkur district, India
}

\section{Sanath Kumar Gurram Krishnamurthy ${ }^{1 *}$, Basavaraj Poojar ${ }^{2}$, Sharath Burugina Nagaraja $^{3}$}

\author{
${ }^{1}$ Department of Health and Family, Tumkur District, Karnataka, India \\ ${ }^{2}$ Department of Pharmacology, Kasturba Medical College, Mangalore, Manipal Academy of Higher Education, Manipal, \\ Karnataka, India \\ ${ }^{3}$ Department of Community Medicine, ESIC Medical College and PGIMSR, Bengaluru, Karnataka, India
}

Received: 01 June 2021

Accepted: 15 June 2021

\section{*Correspondence:}

Dr. Sanath Kumar Gurram Krishnamurthy,

Email:dr.gurumsanath@gmail.com

Copyright: (C) the author(s), publisher and licensee Medip Academy. This is an open-access article distributed under the terms of the Creative Commons Attribution Non-Commercial License, which permits unrestricted non-commercial use, distribution, and reproduction in any medium, provided the original work is properly cited.

\begin{abstract}
Background: the nation-wide lockdown due global pandemic has disrupted a vital strategic intervention resulting in overall $60 \%$ decrease in presumptive and diagnostic TB cases during the lockdown period.

Methods: A discrete choice experimental (DCE) exploratory operational research conducted during March to May 2020.

Results: Health care services were affected $25 \%$ reduction in the outpatient department (OPD) in comparison to the previous year the same period. A gradual reduction in negative sputum cases undergoing chest radiography from 54\% to $14 \%$. Due to restricted movement LPA tests have been reduced $25 \%$ among the diagnosed TB cases, and private referrals to cartridge based nucleic acid amplification test (CBNAAT) services were reduced to $20 \%$.

Conclusions: Health services, including national programmes to combat TB, need to be actively engaged in ensuring an effective and rapid response to COVID-19 while ensuring that TB services are maintained. While experience on COVID-19 infection in TB patients remains limited, it is anticipated that people ill with both TB and COVID-19 may have poorer treatment outcomes, especially if TB treatment is interrupted. TB patients should take precautions as advised by health authorities to be protected from COVID-19 and continue their TB treatment as prescribed.
\end{abstract}

Keywords: National tuberculosis elimination program, COVID-19, Pandemic, Tuberculosis

\section{INTRODUCTION}

India had made significant advancement till March $24^{\text {th }}$ 2020 towards the goal of end TB elimination and then the nation-wide lockdown due global pandemic has disrupted a vital strategic interventions resulting in overall $60 \%$ decrease in presumptive and diagnostic TB cases during the lockdown period. ${ }^{1}$ This may not only impact on morbidity and mortality due to TB, also enhance the active transmission in household contact. The COVID-19 pandemic has placed un-precedented demands and pressure on the health system.

\section{Indian healthcare delivery system}

The national health mission (NHM) is a major instrument of financing and support to the States to strengthen public health systems and healthcare delivery. The private sector provides majority of secondary, tertiary and quaternary care institutions with a major concentration in metros, tier I and tier II cities. ${ }^{2}$ Health facilities and workforce are diverted and assigned a wide variety of activities related to controlling the outbreak hence, the present study is designed to determine the implications of COVID-19 on tuberculosis diseases control strategies. This is a critical 
requirement for evaluating the effectiveness of preventive interventions and for tracking progress.

\section{METHODS}

The study was conducted in the Tumkur district administration of Karnataka, India, from March 2020 to May 2020.

\section{Study design}

The present study was a DCE exploratory operational research. ${ }^{3}$

Qualitative pretested open-ended questions were used to collect free-text comments from peripheral health professionals. Followed by qualitative data think-aloud conceptual basis virtual one to one telephonic conversation was done to collect the data, by predefined data variables and end of conversation debriefing questions, and the answers were done to overcome the recall bias. ${ }^{4}$

\section{Settings}

The study was conducted at the Tumkur district of Karnataka (2.9 million) from March 2020 to August 2020. The district was implementing PMDT since 2011.5 For national tuberculosis elimination program (NTEP) implementation, the district is further sub-divided into tuberculosis units (TU; for every 2.5 million population). The program has established one designated microscopy center (DMC) for every 0.1 million population where the laboratory technicians are trained for sputum smear examination, and the laboratories are quality assured. The district has primary health centers (PHC) for every 0.03 million population, and the DMCs are located at one of these PHCs. The district of Tumkur has 12 TUs with 34 DMCs and 3 CBNAAT sites, the district did not have any DST sites, and the samples were transported to the places outside the district (Nearest DST lab Bangalore).

The qualitative data were obtained from the key informant interviews (KIIs) of seven national tuberculosis elimination program staff of different cadres and five paramedical staff of different cadres involved in national tuberculosis elimination program implementation. The KIIs were conducted through virtual mode in local vernacular language (Kannada) at a time and place convenient to them after taking a prior appointment. Each interview lasted for 8-10 minutes, and all the participants were purposively selected.

\section{Study population, sources of data and data collection}

The data were extracted from the laboratory registers maintained at DMCs drug sensitivity test (DST) registers at CBNAAT sites during March 2020 through the May 2020 .

\section{Data variables}

Quantitative: The data on sputum smear results, date of diagnosis of drug-sensitive TB, the date referred for DRTB test, date of pre-treatment evaluation was extracted from registers and reports. (Required data need to be added).

Qualitative: The pandemic challenges on routine care of NTEP and corrective measures, public health initiatives were taken during pandemic were collected from KII of health care professionals.

\section{Data analysis}

The data was analysed using EpiData analysis (V2.2.2.2.182 Odense, Denmark). Descriptive statistics were tabulated in the form of absolute numbers, and differences in percentage. Manual descriptive thematic analysis was performed for qualitative data.

\section{Ethics approval}

Ethics approval was obtained from the institutional ethics committee of the ESIC medical college and PGIMSR, Bangalore, Karnataka, India. The study involves the record review; a waiver for informed consent was obtained from the ethics committees. Virtual mode informed oral consent was obtained from stakeholder before interviews.

\section{RESULTS}

In ongoing pandemic $\mathrm{TB}$, health care services were affected $25 \%$ reduction in the outpatient department (OPD) in comparison to the previous year the same period (i.e., March-May 2019). In all the institutions' PHI's, CHC's, THs, and in DH. Table 1 shows in the prepandemic total of 6207 presumptive TB cases were examined in the COVID-19 situation overall $56 \%$ (-2705) presumptive cases were identified. There was a gradual reduction in negative sputum cases undergoing chest radiography from $54 \%$ to $14 \%$, but Cough, fever, and cold cases were not referred to DMC. These cases were considered as a suspected COVID infection. Moreover, throat swabs were collected in that health care setup overall $23 \%$ case were severe acute respiratory illness (SARI) samples were confirmed/presumptive based on COVID test result and due to restricted movement LPA tests have been reduced $25 \%$ among the diagnosed TB cases, and private referrals to CBNAAT services were reduced to $20 \%$. In the diagnostic services of the study district.

In Table 2 comparison to the previous year (i.e., March, April 2019), lost-to-fallow and mortality due to TB show a variation in numbers dramatically lost to follow up cases among the diagnosed cases, differing from 9 to $13 \%$. Mortality rate shows significant variation begins with nine deaths in march in which it comes to 1 , but in April, it shows restricted movements might influence 16 deaths due to lockdown, and social anxiety was more evident in the study's provenance. 
Table 1: Comparison of the NTEP diagnostic services during a pandemic pre-COVID/during COVID in Tumkur district, Karnataka March to May 2019-20.

\begin{tabular}{|lclll|}
\hline $\begin{array}{l}\text { Presumptive TB case examination } \\
\text { Diagnostic }\end{array}$ & Pre-COVID & $\begin{array}{l}\text { During } \\
\text { COVII }\end{array}$ & $\begin{array}{l}\text { Change } \\
\text { absolute no. }\end{array}$ & Change \% \\
\hline March & 2066 & 2249 & +183 & 108.85 \\
\hline April & 2123 & 876 & -1247 & 41.26 \\
\hline May & 2018 & 377 & -1641 & 18.68 \\
\hline Total & 6207 & 3502 & -2705 & 56.42 \\
\hline Confirmed +ve subjects & & & & \\
\hline March & 227 & 90 & -137 & 39.64 \\
\hline April & 222 & 88 & -134 & 39.63 \\
\hline May & 221 & 33 & -188 & 14.93 \\
\hline Total & 670 & 211 & -459 & 31.49 \\
\hline Sputum negative cases tested for chest X-ray & & & & \\
\hline March & 385 & 209 & -176 & 54.28 \\
\hline April & 410 & 110 & -300 & 26.82 \\
\hline May & 459 & 65 & -394 & 30.16 \\
\hline Total & 1254 & 384 & -870 & \\
\hline Sputum samples of SARI cases referred to CBNAAT & & & 22.22 \\
\hline April & 27 & 6 & -21 & 25.00 \\
\hline May & 24 & 6 & -18 & 23.52 \\
\hline Total & 51 & 12 & -39 & 6.94 \\
\hline Sputum samples of ILI cases referred to CBNAAT & & & 3.89 \\
\hline April & 72 & 5 & -67 & 5.36 \\
\hline May & 77 & 3 & -74 & -141 \\
\hline Total & 149 & 8 & & \\
\hline
\end{tabular}

Table 2: Comparison of the NTEP treatment services during pandemic pre-COVID/during COVID in Tumkur district, Karnataka March to May 2019-20.

\begin{tabular}{|lllll|}
\hline Variables & $\begin{array}{l}\text { Pre-COVID } \\
\text { Lost to follow up cases among the diagnosed cases }\end{array}$ & During COVID & $\begin{array}{l}\text { Change } \\
\text { absolute no. }\end{array}$ & Change \% \\
\hline March & 22 & 2 & -20 & 9.09 \\
\hline April & 16 & 2 & -14 & 12.25 \\
\hline May & 15 & 2 & -13 & 13.33 \\
\hline Total & 53 & 6 & -47 & 11.32 \\
\hline Deaths among diagnosed TB cases & & & & \\
\hline March & 33 & 9 & -24 & 27.27 \\
\hline April & 35 & 16 & -19 & 45.71 \\
\hline May & 31 & 1 & -30 & 3.22 \\
\hline Total & 99 & 26 & -73 & 26.26 \\
\hline
\end{tabular}

\section{DISCUSSION}

\section{Qualitative}

The challenges perceived by the health professionals and care providers in COVID-19 pandemic and corrective measures taken to the implementation of CDT guidelines were broadly categorized as 1) Diagnostic services, 2) treatment services, 3) Public health action: (1a) challenges are diagnostic services and (1b) Corrective measures taken for diagnostic services (2a) Treatment services and its implementation steps.

\section{Challenges is diagnostic services}

DMC technicians are reluctant to do smear for AFB due to the COVID pandemic and lacking of PPE kits, masks, and other safety equipment and till the end of March month. The ASHA's, AW, and other health care professionals were surveying in COVID affected areas and adjoining buffer zones, and they are reluctant to collect the sputum samples. Furthermore, they expressed their apprehension due non defined guidelines in collecting samples from home care services, in turn, they refer ILI (Influenza like illness) and SARI (severe acute respiratory illness) to 
health care setup (government hospitals) for throat swab testing.

Due to the applicability of restricted movements in the nation, the courier services were affected, transporting sputum samples to IRL labs for LPA testing were made available through alternative mode.

Private practitioner's (PP) health care setup was closed according to the government guidelines during this period. From March 1 $1^{\text {st }}, 2020$ onwards 50\% through the first case was identified March $25^{\text {th }}$, of the PP's, are opened the hospitals and clinics were not seeing the fever and cough cases directly referred to public health institutions.

Corrective measures: have taken to identify presumptive cases and case detection of TB pandemic with following standard operating guidelines (SOP) for DMC and sputum collection center were prepared on par with CTD guidelines, to take safety measures during corona pandemic (Annexure 1).

The sample handler PPE kit, masks, face shields, gloves, sanitizers, and sodium hypochlorite solutions were provided, including DMC's. Re-routed all the collected samples from the DMC's to CBNAAT sites (3 CBNAAT sites) and were transported through the vehicles which were carrying COVID-19 specimen to district headquarters in the separate package by following standard practice along with demarcation remarks to differentiate samples from COVID like covering with cupboard box, mentioning postal address, defining contact person details along with a mobile number. The vehicle carrying COVID-19 specimen to state headquarters and sputum samples was also sent to ILR, Bangalore, for the LPA.

ILI and SARI cases line list was prepared during throat swab collection, and after getting COVID negative results, sputum samples were collected and transported to the CBNAAT site. Subjecting to x-ray after COVID-19 negative ILI cases, it was a daunting task to bring back patients due to the non-availability of transportation as there was restricted movement, only town area patients are subjected for a chest x-ray. All SARI cases were admitted in the district hospital to collected sputum samples, and samples were transported to the CBNAAT site through TBHV's.

\section{Treatment services and its implementation steps}

As per CTD guidelines issued in March concerning the advance distribution of fixed-dose combination (FDC) for two months to ongoing treatment cases, we from the Tumkur district immediately into action. Stocks were made available adequately during the lockdown period. ${ }^{6}$ Peripheral healthcare workers and community health workers (STS, STLS, and TBHV's) were visiting TB patients' homes and providing home care services. STLS were sensitized through phone calls and virtual meetings to collect follow-up sputum samples from the patient's house regularly, and samples were transported to respective DMCs for follow-up smear examination. During the community visit, NTEP staff were doing primary contact tracing of households of ongoing treatment subjects. NTEP staff was ensured monthly DBT transfer to TB patients during the lockdown period and by confirming through a phone call to the subject whether the amount was credited to their account or not. Though case detection was low in lockdown period through the utilization of contact tracing mode case detection, existing subjects were followed up to enhance detection and monitoring services of NTEP.

By effortful assurance, community health care workers were made to take care of their health to ongoing treatment subjects, along with strict adherence to the home quarantine. Furthermore, sanitized to self-vigilant about day to day activates of the family members and educated on health care seeking behavior during pandemic lockdown period since March, it is an ongoing process, with these local efforts bacilli transmission might reduce and diseases conversion rate will decrease in coming years. We are hoping the TB disease transmission dynamic will significantly reduce by following national pandemic control instruction like wearing masks, social distancing, and practicing cough etiquette, which was laid down by central governance.by and large, we are progressing towards the eradication of tuberculosis.

\section{Public health action has done during corona pandemic}

From April onwards, NTEP staff were preparing list 50 subjects daily for tele-healthcare services such as a telephonic call to the subject on COVID symptoms like fever, cough, cold, throat pain, and breathlessness, which are similar to TB symptoms and made awareness to the healthcare-seeking behavior during a pandemic. Efforts were made to install the "Arogya Sethu" application to the subject mobile by the community health workers and educating their uses for COVID related information each individual in there defined health care setup. With the association of NTEP, staff fund rose to distribute food grains and groceries worth of 500 rupees to each needy subject. Fund was pooled from salary on voluntary willingness in societal concern, and it includes district program manager salary.

The district program manager conducted virtual meetings through social media platforms to create awareness about pharmacovigilance and usage of adverse drug reaction reporting. Pharmacovigilance program (PvPI) application to the community health worker by them sensitized TB infected subjects regarding side effects (Adverse drug reaction), and they can report directly to the national tollfree number. With these efforts, we are heading towards minimizing under-reporting of adverse drug reaction (ADR) and trying to contribute to establishing evidential documentation of adverse effects. 


\section{CONCLUSION}

Health services, including national programmes to combat $\mathrm{TB}$, need to be actively engaged in ensuring an effective and rapid response to COVID-19 while ensuring that TB services are maintained. While experience on COVID-19 infection in TB patients remains limited, it is anticipated that people ill with both TB and COVID-19 may have poorer treatment outcomes, especially if TB treatment is interrupted. TB patients should take precautions as advised by health authorities to be protected from COVID-19 and continue their TB treatment as prescribed.

Funding: No funding sources

Conflict of interest: None declared

Ethical approval: The study was approved by the Institutional Ethics Committee

\section{REFERENCES}

1. Jain VK, Iyengar KP, Samy DA, Vaishya R. Tuberculosis in the era of COVID-19 in India. Diabetes Metabolic Syndrome: Clin Rese Reviews. 2020;14(5):1439-43.

2. ICMR, PHFI and I. Karnataka: Disease Burden Profile, 1990-2016. India Heal Nation's States-India
State-Level Dis Burd Initiat New Delhi. 2017;5-8.

3. Soekhai V, de Bekker-Grob EW, Ellis AR, Vass CM. Discrete Choice Experiments in Health Economics: Past, Present and Future. Pharmacoeconomics. 2019;37(2):201-26.

4. Lavrakas P. Interviewer Debriefing. In: Encyclopedia of Survey Research Methods. Sage Publications, Inc. 2013.

5. Tumkur District Population (2019/2020), District Talukas List, Karnataka. Available from: http://www.indiagrowing.com/Karnataka/Tumkur_D istrict. Accessed on 2020 Jun 7.

6. Government of India. Advisory from Central TB Division. Available at: https://tbcindia.gov.in/ WriteReadData/AdvisoryDDStatesUTs.pdf. Accessed on 2020 Jun 7.

Cite this article as: Krishnamurthy SKG, Poojar B, Nagaraja SB. Effects of COVID-19 on national tuberculosis elimination programme strategies during March to May 2020 on Tumkur district, India. Int J Basic Clin Pharmacol 2021;10:937-42. 


\section{Annexure}

\section{Standard operating procedures for DMC and sputum collection centers}

\section{Good laboratory practices and universal safety precautions}

1. Washing hands with soap and water after collecting, receiving and testing specimens.

2. No eatables and drinking braveries allowed inside the laboratory.

3. Access will be restricted inside the laboratory other than lab technologist.

4. If more than one technician posted is posted in laboratory, distance between the personnel must be maintained.

5. The working surfaces must be made free of organic matter with the help of $1 \%$ sodium hypochlorite and large amount of folded absorbent paper. This should be followed by disinfection of work surfaces using $1 \%$ sodium hypochlorite, allowing 10-20 minutes contact time with sodium hypochlorite.

6. Work should be started only after the surface is completely dried, this exercise should be repeated twice in a day (before the start and after completion of the day's work).

7. Laboratory technologist must wear the following personal protective equipment (PPE) while handling specimens:

a. Disposal N95 respirators (mask), b. Disposable apron/surgical gown/normal apron covered with plastic sheet (disposable/autoclavable/disinfected with hypochlorite), 